\title{
Application of resuscitative endovascular balloon occlusion in post-transplant mycotic hepatic artery pseudoaneurysm rupture in the setting of Aspergillus Constellatus bacteremia
}

\author{
Krista Stephenson ${ }^{1}$, Kyle Kalkwarf ${ }^{2}$, and Emmanouil Giorgakis ${ }^{1}$ \\ ${ }^{1}$ Division of Solid Organ Transplantation, Department of Surgery, UAMS Medical Center, \\ ${ }^{2}$ Division of Trauma and Critical Care Surgery, Department of Surgery, UAMS Medical Center, Little Rock, AR, USA
}

\begin{abstract}
Hepatic artery pseudoaneurysm (HAP) is a rare, highly morbid and frequently fatal complication of liver transplantation. Most are a mycotic mediated weakness of the arterial wall, with associated bacterial or fungal infection of ascitic fluid. As it is usually asymptomatic prior to rupture, the majority present in acute hemorrhagic shock and dire extremis. Resuscitative endovascular balloon occlusion (REBOA) was initially developed for the management of noncompressible hemorrhagic shock in trauma; however, remains underutilized and understudied in the non-trauma setting. We present the case of a mycotic hepatic artery pseudoaneurysm rupture due to Streptococcus constellatus and Klebsiella pneumoniae post directed donor orthoptic liver transplant, in which REBOA was employed in the setting of impending exsanguination as a bridge to definitive surgical intervention. Although this patient passed away of multiorgan system failure prior to re-transplant, this case demonstrates the importance of a heightened suspicion of this devastating complication, especially in the setting of bilioenteric reconstruction and perihepatic fluid collection, as well as the benefit of utilizing resuscitative techniques such as REBOA prior to definitive surgical or endovascular therapy to mitigate the high morbidity and mortality of this condition. (Ann Hepatobiliary Pancreat Surg 2021;25:126-131)
\end{abstract}

Key Words: Hepatic artery pseudoaneurysm; Streptococcus constellatus; REBOA; Resuscitative endovascular balloon occlusion; Liver transplantation

\section{INTRODUCTION}

Hepatic artery (HA) pseudoaneurysm is a rare, highly morbid and frequently fatal complication of liver transplantation (LT), with a reported incidence ranging from $0.01-2.5 \%$ and mortality rate of $42-100 \% .^{1-4}$ The majority present within the first two months post-transplant, and remain asymptomatic prior to rupture, causing most (50-85\%) to present in acute hemorrhagic shock from gastrointestinal or intraperitoneal hemorrhage. ${ }^{1-4}$ The risk is significantly increased by bacterial or fungal colonization of perihepatic fluid due to a resultant mycotic aneurysmal weakening of the arterial wall. This is evidenced by positive ascitic cultures, with reported ascitic fluid colonization in $75-83 \%$ at time of diagnosis. ${ }^{2-4}$ Hepatic artery pseudoaneurysms have also repeatedly demonstrated to be associated with bilio-enteric anastomosis, reported in 43$77 \%,{ }^{2-4}$ as well as postoperative bile leak, seen in $15-44 \%,{ }^{3,4}$ likely through the same mechanism. Due to its acute presentation and associated high morbidity and mortality, survival is dependent upon prompt diagnosis and treatment.

Resuscitative endovascular balloon occlusion of the aorta (REBOA) is an intervention initially developed for treatment of noncompressible hemorrhagic shock in trauma as a temporizing measure prior to definitive hemorrhage control (Fig. 1)..$^{5}$ It has been largely employed in this indication; however, remains largely underutilized and understudied in other settings. ${ }^{6,7}$ The procedure involves cannulation of the common femoral artery, insertion of an introducer sheath with Seldinger technique over a guidewire, and placement of the device by either landmark or radiologic guidance into an aortic zone. Zone 1 inflation,

Received: July 24, 2020; Revised: September 23, 2020; Accepted: September 27, 2020

Corresponding author: Krista Stephenson

Division of Solid Organ Transplantation, Department of Surgery, UAMS Medical Center, 4301 W Markham St. - Slot \#520, Little Rock, AR 72205, USA

Tel: +1-501-364-2832, Fax: +1-501-686-6375, E-mail: KStephenson2@uams.edu

Copyright (C) 2021 by The Korean Association of Hepato-Biliary-Pancreatic Surgery

This is an Open Access article distributed under the terms of the Creative Commons Attribution Non-Commercial License (http://creativecommons.org/ licenses/by-nc/4.0) which permits unrestricted non-commercial use, distribution, and reproduction in any medium, provided the original work is properly cited. Annals of Hepato-Biliary-Pancreatic Surgery - pISSN: 2508-5778 - elSSN: 2508-5859 


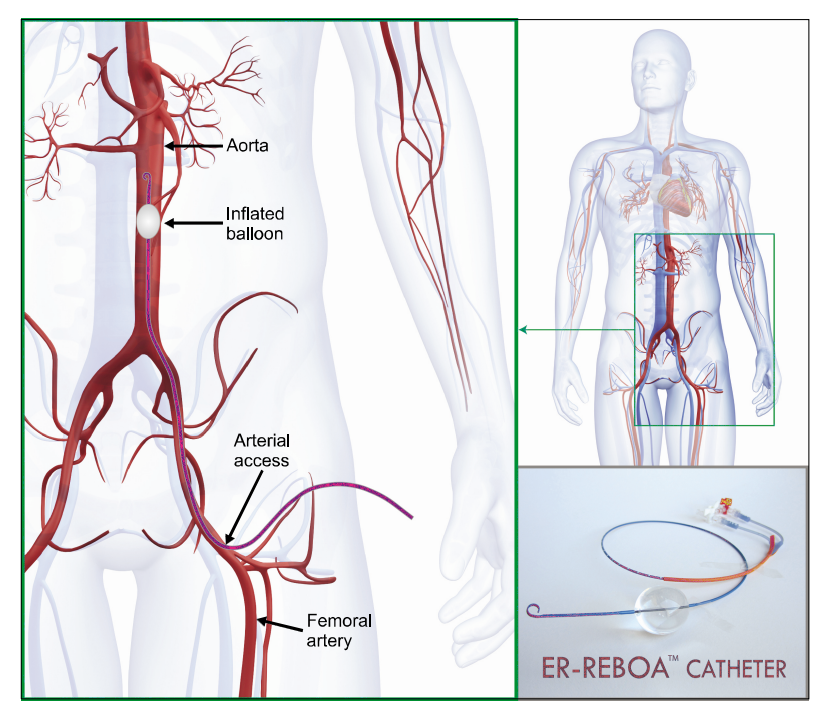

Fig. 1. REBOA catheter placement involves cannulation of the common femoral artery, insertion of an introducer sheath with Seldinger technique over a guidewire, and placement of the device by either landmark or radiologic guidance into an aortic zone. Zone 1 inflation, located between the left subclavian artery and the celiac trunk, is used for abdominal hemorrhage control.

located between the left subclavian artery and the celiac trunk, is used for abdominal hemorrhage control. Balloon occlusion of the aorta at this level allows for temporary hemorrhage control and blood pressure elevation proximal to the balloon, resulting in increased in cerebral and coronary circulation as a bridge to definitive endovascular or surgical treatment. ${ }^{7}$

We present a case of a mycotic HA pseudoaneurysm rupture following orthotopic LT in the setting of Streptococcus Constellatus bacteremia, with the use of REBOA as a resuscitating bringing maneuver prior to surgical exploration and HA ligation.

\section{CASE}

The patient was a 64-year-old male who underwent a LT for end-stage liver disease due to secondary biliary cirrhosis and non-alcoholic steatohepatitis. He had a history of a robotic cholecystectomy, complicated by a hepatic duct leak, requiring open choledochojejunostomy. He subsequently developed persistently elevated liver function tests and underwent magnetic resonance cholangiopancreatography, that revealed a choledochojejunostomy stricture and intrahepatic biliary tree dilation. A liver biopsy was consistent with cirrhosis attributed to chronic biliary obstruction and superimposed non-alcoholic steatohepatitis.

The patient underwent a LT receiving an excellent quality graft from a brain-dead donor. The procedure was tedious due to extensive adhesions from his previous surgeries. Graft implantation was with an extended two-hepatic vein piggy-back technique, portal vein to portal vein anastomosis, common HA to common HA anastomosis at the confluence of the gastroduodenal artery (GDA), as well as reconstruction of a small accessory right HA to the donor GDA. Biliary reconstruction was performed using the biliary limb of his previous choledochojejunostomy. Per protocol, a steroid taper was performed, Myfortic and Tacrolimus immunosuppression was initiated, and he received Micafungin, Valcyte, and Bactrim prophylaxis.

His postoperative course was complicated by thrombus of the accessory right HA, discovered on post-operative day 1 on routine Doppler ultrasound; however, on computed tomography angiography the common HA remained patent, providing adequate collateral arterial flow to the right lobe. It was felt the risk of procedural intervention would not outweigh the benefits, due to a high re-thrombosis risk of the re-reconstructed vessel due to its small caliber, plus the necessity for a biliary anastomosis takedown, in addition to the risk of damage to the main HA anastomosis should a surgical revision be attempted. Therefore, the patient was started on therapeutic low molecular weight heparin to prevent propagation of the thrombus. This resulted in a perihepatic hematoma for which a percutaneous drain was placed. Drain fluid cultures were negative and drain output remained nonbilious. He remained on therapeutic anticoagulation and was transitioned to Apixaban at time of discharge on post-operative day 8 . He was doing well and progressing as expected on routine follow-up appointments, and the perihepatic drain was removed on post-operative day 10 .

Three weeks post-transplant (post-operative day 22), he was brought to the emergency department due to sudden onset, severe, right upper quadrant abdominal pain and lightheadedness. Upon arrival, he was hemodynamically unstable, tachycardic and hypotensive, with noted abdominal distension. Massive transfusion protocol was activated and resuscitation was quickly initiated, along with sepsis protocol. Labs revealed a severe lactic acidosis with a bicarbonate of $8 \mathrm{mmol} / \mathrm{L}$ and lactate of $9.8 \mathrm{mmol} / \mathrm{L}$, acute blood loss anemia with a hemoglobin of $6.5 \mathrm{~g} / \mathrm{dl}$, acute kidney injury 


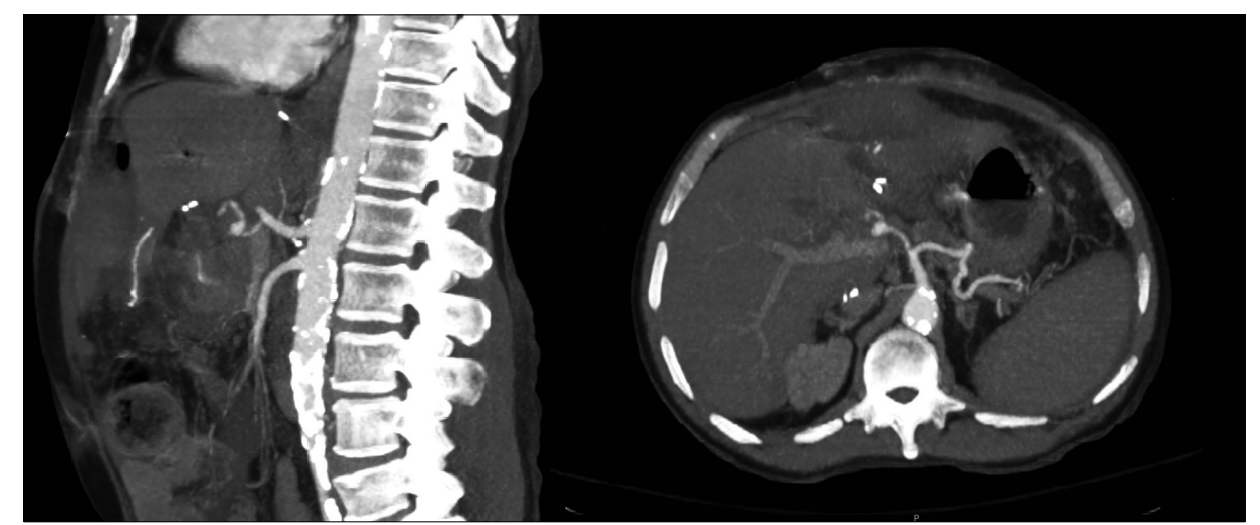

Fig. 2. Computed tomography revealing a main HA pseudoaneurysm with active extravasation and an associated hemoperitoneum. with a creatinine of $3.1 \mathrm{mg} / \mathrm{dl}$, as well as worsening liver function tests. He responded transiently to blood product resuscitation, and computed tomography was performed, which revealed a main HA pseudoaneurysm with active extravasation and an associated moderate amount of hemoperitoneum (Fig. 2).

The decision was made to proceed with endovascular therapy; however, en route to the interventional radiology (IR) suite, he sustained an acute decompensation with a decrease in mean arterial pressure to $30-40 \mathrm{mmHg}$, unresponsive to continued blood product resuscitation. On transitioning to the IR table, he went into pulseless electrical activity (PEA). Advanced cardiac life support (ACLS) was initiated, intubation was performed, and a right femoral arterial line was exchanged to a REBOA sheath, inflated at zone 1 with ongoing MTP resuscitation. After cardiopulmonary resuscitation with the REOBOA inflated, his spontaneous cardiac activity recovered, allowing for emergency transport to the operating room.

A rapid laparotomy was performed with the REBOA remaining inflated. Open abdominal opening, a large amount of fresh blood clot and hematoma to the hepatic hilum was evident. There was no evidence of biloma, bile leak, enteric leak, or abscess formation; however, the hepatic allograft appeared ischemic due to cessation of abdominal aortic perfusion, as the REBOA had been at total occlusion 22 minutes prior to laparotomy. After rapid identification and control of the recipient HA, the REBOA was slowly deflated and intraabdominal aorta was allowed to re-perfuse, resulting in a severe reperfusion injury with recurrent PEA. The REBOA was re-inflated to occlusion and ACLS was again initiated, with resumption of pulse, resulting in an additional 5 minutes of aortic occlusion. At this time, the REBOA was deflated and the graft vasculature was interrogated. The donor proper $\mathrm{HA}$ and its branches were noted to be thrombosed and damaged up to the entry of the liver; therefore, not amenable to reconstruction. The anterior wall of the donor HA had ruptured and disintegrated on fingertip manipulation, indicative of advanced mycotic change. The recipient HA stump was ligated and an open abdomen negative pressure therapy (ABTHERA) was placed. The patient was transferred to the surgical intensive care unit for further resuscitation in dire extremis. Continuous renal replacement therapy was initiated and goal directed resuscitation continued for his refractory septic shock, severe acidosis, electrolyte abnormalities, and coagulopathy following the hepatic ischemic and hemorrhagic insult, with a goal to stabilize and temporize in anticipation of enlisting for an emergency re-transplant as a status $1 \mathrm{~A}$.

His condition continued to decline in spite of continued resuscitation, due to the combined hit of refractory septic shock with multiorgan failure and allograft non-function. Approximately 14 hours postoperatively, he became bradycardic and pulseless. At family's request, further resuscitative efforts were discontinued, and he was pronounced deceased. Postmortem preoperative and intraoperative microbiology results were obtained, revealing Streptococcus Constellatus bacteremia as well as Strep Constellatus and Klebsiella Pneumoniae colonization of the evacuated hematoma.

\section{DISCUSSION}

Mycotic HA pseudoaneurysm remains a highly morbid and frequently lethal post-transplant complication, with an 
estimated incidence of $0.01-2.5 \%$ based upon a limited number of case series, which likely underrepresent those with fatal outcome. ${ }^{1-4}$ Previous reports have shown that infectious microorganisms are implicated in their development. Imaging findings in addition to possible treatment options of non-ruptured cases have been well described. However, if the pseudoaneurysm remains unrecognized prior to rupture, immediate surgery becomes mandatory. Multiple risk factors, including bilio-enteric reconstruction, bile leak, and ascitic fluid colonization have been established, ${ }^{2-4}$ and recent publications have suggested possible benefit of a surveillance protocol to detect this complication prior to rupture, especially in those with known risk factors. ${ }^{4,8}$

HA mycotic aneurysm formation has been previously associated with gastrointestinal flora, including Escherichia coli and Enterococcus spp as well as opportunistic fungal infections as Candida Glabrata and Pseudomonas., ${ }^{2,9}$ Most reported cases are associated with local bacterial contamination, commonly with bile leak. ${ }^{3,4}$ In the case presented, there was no biloma formation; however, cultures of the evacuated hematoma around the ruptured aneurysm were positive for Streptococcus Constellatus and Klebsiella Pneumoniae. In addition, Streptococcus Constellatus bacteremia was also present, evident in all obtained blood cultures at the time of his presentation. Streptococcus Constellatus is a non-spore-forming gram-positive coccus belonging to the streptococcus anginosus group (formerly Streptococcus Mileri group). While it is present in oral, respiratory, gastrointestinal and genitourinary floras, it is usually associated with suppurative thoracic infections. ${ }^{10,11}$ While not previously thought to cause mycotic aneurysms, there have been isolated case reports, including a pulmonary artery pseudoaneurysm ${ }^{10}$ as well as an ascending aortic aneurysm in lymphoma patient ${ }^{11}$ in which St. Constellatus was implicated. Klebsiella pneumonia, also isolated from the evacuated hematoma, has been previously associated with a mycotic HA rupture after transplant in a single case report $^{12}$ and additionally has been discovered in scattered cases of mycotic aortic aneurysms with associated bacteremia.

As most mycotic HA pseudoaneurysms present in extremis, prompt diagnosis of pseudoaneurysm prior to rupture is key. Unfortunately, mycotic HA pseudoaneurysm rupture prior to endovascular or surgical intervention is not uncommon and is nearly universally fatal, as exsanguination can ensue in a matter of minutes. In this case,
REBOA was expeditiously employed, guide-wiring through an already placed arterial line, which allowed for transitory hemorrhage control and response to MTP. Its use enabled return of spontaneous cardiac activity and allowed for emergency laparotomy and surgical control of the HA bleed; however, at the expense of abdominal visceral ischemia.

In recent years, there have been several case reports and case series evaluating the use of REBOA in nontraumatic hemorrhagic shock, including use in gastrointestinal hemorrhage, solid organ injury, visceral aneurysms, abdominal aortic aneurysms, and post-surgical abdominal hemorrhage. The literature has persistently demonstrated improvement in proximal blood pressure and prevention of exsanguination, but has shown varied morbidity incidence and survival benefit in nontraumatic hemorrhage. ${ }^{6,7}$ Known procedural complications include iatrogenic vascular injury with aortic dissection, rupture, and peripheral ischemia. More importantly, aortic occlusion results in a time-dependent end-organ ischemia and inflammatory response. Studies have shown an unavoidable reperfusion injury at inflation times greater than $30 \mathrm{mi}$ nutes, with a resultant hyperkalemia, acidosis, and associated risk of hypotension and cardiac collapse upon REBOA deflation. ${ }^{6}$ This injury is also evidenced by a prolonged elevation intrahepatic lactate and glycerol, as well as high plasma concentrations of liver, kidney, pancreatic, and skeletal muscle enzymes that contribute to multiorgan system failure and mortality. ${ }^{13}$ Notably, this response may be decreased with prompt definitive hemorrhage control, enabling a short inflation time, and may also be blunted with partial balloon occlusion. ${ }^{14}$

In the case presented, REBOA was employed in the setting of impending exsanguination as an adjunct to enable definitive hemorrhage control. Even though the total aortic occlusion period was short, balloon deflation was followed by a massive reperfusion injury, resulting in intraoperative cardiac arrest despite hemorrhage control. And although return of spontaneous cardiac activity and pulse was regained, the reperfusion injury, compounded by graft nonfunction and a refractory vasodilatory septic shock caused by Streptococcus Constellatus bacteremia, resulted in multiorgan failure, severe electrolyte derangement, acidosis, and coagulopathy from which he never recovered despite successful bleeding control and hepatic artery ligation. 
There are many described treatment options for HA pseudoaneurysm repair. Surgical options include HA excision with immediate surgical revascularization, with or without the use of an aortic jump graft, and HA ligation. HA revascularization has a lower mortality as well as an improved graft salvage rate, but requires remaining arterial integrity and should not be performed in the presence of arterial infection. ${ }^{3,415}$ When arterial reconstruction proves unfeasible, as in the case presented, HA ligation can be performed; however, is associated with a high early mortality rate $(28-85 \%)$ as well as frequent loss of graft viability requiring re-transplant. ${ }^{3,4}$ Although, surprisingly HA ligation following HA rupture does not always necessitate re-transplantation. ${ }^{16}$ In recent years, minimally invasive percutaneous endovascular interventions have been increasingly utilized, including coil embolization ${ }^{4,17}$ and thrombin injection ${ }^{18}$ as well as insertion of flow diverting bare stents and stent grafts. ${ }^{4,9,19}$ Endovascular techniques have the advantage of being both diagnostic and therapeutic, and have been found to achieve more rapid bleeding control, shorter hospital stay, lower transfusion requirement, with significantly decreased morbidity and mortality rates. However, there remain multiple anatomic contraindications for endovascular treatment and it must be performed prior to complete pseudoaneurysm rupture. ${ }^{19,20}$

\section{CONCLUSION}

The presented case is notable in several ways. It identifies Streptococcus Constellatus as another possible cause of mycotic aneurysm formation after liver transplantation, posing that the presence of Streptococcus Constellatus bacteremia should raise suspicion for arterial mycosis in the post-transplant setting. Additionally, it demonstrates the application of REBOA in the commonly fatal setting of complete HA pseudoaneurysm rupture. Although the patient succumbed to multiorgan system failure and septic shock, we hope presentation of the techniques employed in his care spawn further discussion and research, resulting in improved diagnosis, treatment, and survival in this rare and devastating complication. While the use of REBOA did not alter the tragic outcome on this occasion, the authors believe that its addition to the resuscitative armamentarium of HA pseudoaneurysm rupture may afford the extra time desperately needed to halt catastrophic exsan- guination, and ultimately, save the post-transplant patient's life.

\section{ORCID}

Krista Stephenson: https://orcid.org/0000-0001-7591-6097

Kyle Kalkwarf: https://orcid.org/0000-0003-1693-4935

Emmanouil Giorgakis: https://orcid.org/0000-0002-5019-5497

\section{AUTHOR CONTRIBUTIONS}

Writing - original draft: KS. Writing - review \& editing: $\mathrm{KS}, \mathrm{KK}, \mathrm{EG}$.

\section{REFERENCES}

1. Langnas AN, Marujo W, Stratta RJ, Wood RP, Shaw BW Jr. Vascular complications after orthotopic liver transplantation. Am J Surg 1991;161:76-82; discussion 82-83.

2. Leelaudomlipi S, Bramhall SR, Gunson BK, Candinas D, Buckels JA, McMaster P, et al. Hepatic-artery aneurysm in adult liver transplantation. Transpl Int 2003;16:257-261.

3. Marshall MM, Muiesan P, Srinivasan P, Kane PA, Rela M, Heaton ND, et al. Hepatic artery pseudoaneurysms following liver transplantation: incidence, presenting features and management. Clin Radiol 2001;56:579-587.

4. Volpin E, Pessaux P, Sauvanet A, Sibert A, Kianmanesh R, Durand F, et al. Preservation of the arterial vascularisation after hepatic artery pseudoaneurysm following orthotopic liver transplantation: long-term results. Ann Transplant 2014;19:346-352.

5. Gupta BK, Khaneja SC, Flores L, Eastlick L, Longmore W, Shaftan GW. The role of intra-aortic balloon occlusion in penetrating abdominal trauma. J Trauma 1989;29:861-865.

6. Morrison JJ, Galgon RE, Jansen JO, Cannon JW, Rasmussen TE, Eliason JL. A systematic review of the use of resuscitative endovascular balloon occlusion of the aorta in the management of hemorrhagic shock. J Trauma Acute Care Surg 2016;80:324-334.

7. Ribeiro Júnior MAF, Maurício AD, Costa CTK, Néder PR, Augusto SS, Di-Saverio S, et al. Expanding indications and results for the use of resuscitative endovascular balloon occlusion of the aorta - REBOA. Rev Col Bras Cir 2019;46:e20192334.

8. Frongillo F, Lirosi MC, Nure E, Inchingolo R, Bianco G, Silvestrini $\mathrm{N}$, et al. Diagnosis and management of hepatic artery complications after liver transplantation. Transplant Proc 2015;47: 2150-2155.

9. Thorat A, Lee CF, Wu TH, Pan KT, Chu SY, Chou HS, et al. Endovascular treatment for pseudoaneurysms arising from the hepatic artery after liver transplantation. Asian J Surg 2017;40:227231.

10. Morinaga Y, Yanagihara K, Gyotoku H, Oshima K, Izumikawa $\mathrm{K}$, Yamasaki N, et al. Pulmonary artery pseudoaneurysm caused by Streptococcus constellatus. Int J Infect Dis 2013;17:e1064e1066.

11. Raja NS, Nikolova A, Raudsepp T, Weston-Smith SG, Rumbold A, Shaikh H, et al. Streptococcus constellatus causing mycotic aneurysm in a patient with diffuse large B-cell lymphoma. J Microbiol Infect Dis 2017;7:213-216.

12. Qian Y, Zhang H, Chen X, Zhang J, Xia Q. Graft hepatic artery 
rupture due to carbapenem-resistant Klebsiella pneumoniae infection after liver transplant. Exp Clin Transplant 2020;18:529-532.

13. Sadeghi M, Dogan EM, Karlsson C, Jansson K, Seilitz J, Skoog $\mathrm{P}$, et al. Total resuscitative endovascular balloon occlusion of the aorta causes inflammatory activation and organ damage within 30 minutes of occlusion in normovolemic pigs. BMC Surg 2020; 20:43.

14. Forte DM, Do WS, Weiss JB, Sheldon RR, Kuckelman JP, Eckert MJ, et al. Titrate to equilibrate and not exsanguinate! Characterization and validation of a novel partial resuscitative endovascular balloon occlusion of the aorta catheter in normal and hemorrhagic shock conditions. J Trauma Acute Care Surg 2019;87:1015-1025.

15. Bonham CA, Kapur S, Geller D, Fung JJ, Pinna A. Excision and immediate revascularization for hepatic artery pseudoaneurysm following liver transplantation. Transplant Proc 1999;31:443.

16. Boleslawski E, Bouras AF, Truant S, Liddo G, Herrero A, Badic $\mathrm{B}$, et al. Hepatic artery ligation for arterial rupture following liv- er transplantation: a reasonable option. Am J Transplant 2013;13: 1055-1062.

17. Tulsyan N, Kashyap VS, Greenberg RK, Sarac TP, Clair DG, Pierce G, et al. The endovascular management of visceral artery aneurysms and pseudoaneurysms. J Vasc Surg 2007;45:276-283; discussion 283.

18. Patel JV, Weston MJ, Kessel DO, Prasad R, Toogood GJ, Robertson I. Hepatic artery pseudoaneurysm after liver transplantation: treatment with percutaneous thrombin injection. Transplantation 2003;75:1755-1757.

19. Pedersoli F, Isfort P, Keil S, Goerg F, Zimmermann M, Liebl $\mathrm{M}$, et al. Stentgraft implantation for the treatment of postoperative hepatic artery pseudoaneurysm. Cardiovasc Intervent Radiol 2016;39:575-581.

20. Sachdev U, Baril DT, Ellozy SH, Lookstein RA, Silverberg D, Jacobs TS, et al. Management of aneurysms involving branches of the celiac and superior mesenteric arteries: a comparison of surgical and endovascular therapy. J Vasc Surg 2006;44:718-724. 